\title{
Final Assembly of the Helmholtz-Zentrum Berlin Series-Connected Hybrid Magnet System
}

\author{
Iain R. Dixon, Member, IEEE, Todd A. Adkins, Mark D. Bird, Member, IEEE, Scott T. Bole, \\ Jack Toth, Hartmut Ehmler, Matthias Hoffman, and Peter Smeibidl
}

\begin{abstract}
The final assembly of the Series-Connected Hybrid magnet system for the Helmholtz-Zentrum Berlin for Materials and Energy (HZB) has occurred with the integration of the superconducting cold mass, cryostat, resistive Florida-Bitter coils, and the cryogenic, chilled water, power, and control subsystems. The hybrid magnet consists of a 13-T superconducting $\mathrm{Nb}_{3} \mathrm{Sn} / \mathrm{CICC}$ coil and a set of 12-T resistive, water cooled coils at $4.4 \mathrm{MW}$. Much of the cryostat and cold mass functional requirements were dictated by the electromagnetic interactions between the superconducting and resistive coils. This includes the radial decentering and axial aligning forces from normal operations and a 1.1 MN fault load. The system assembly was an international achievement with the cold mass being completed at the NHMFL in the USA, cryostat to cold mass interfaces made at Criotec Impianti in Italy, and final assembly at the HZB in Germany.
\end{abstract}

Index Terms-Cable-in-conduit conductors, hybrid magnets, $\mathrm{Nb}_{3} \mathrm{Sn}$, superconducting magnets.

\section{INTRODUCTION}

$\mathbf{T}$ HE phases of construction of a hybrid magnet system [1] and facility to produce a strong magnetic field of $25 \mathrm{~T}$ in a $50 \mathrm{~mm}$ - conical bore for neutron scattering experiments at the Helmholtz-Zentrum Berlin (HZB) are over and a magnet commissioning phase has commenced. Once operational, the magnet system will be part of the Extreme Environment Diffractometer to study the structure and dynamics of materials, primarily high temperature superconductors [2]. The new HZB Series-Connected Hybrid magnet constitutes a $47 \%$ increase in field coupled with a $100 \%$ increase in solid-angle over magnets available for neutron scattering.

The primary technical parameters are provided in Table I. The field contributed by the resistive coils has the potential to be increased to $24 \mathrm{~T}$ with an upgrade in the power supply and chilled water systems. The resistive coils [3] are the first to have a conically conforming inner diameter to take advantage of the available space in the conical bore, which is shaped as such to

Manuscript received August 12, 2014; accepted September 29, 2014. Date of publication October 2, 2014; date of current version December 23, 2014. This work was supported in part by the German government, National Science Foundation (award DMR-0603042), and the State of Florida.

I. R. Dixon, T. A. Adkins, M. D. Bird, S. T. Bole, and J. Toth are with the National High Magnetic Field Laboratory, Tallahassee, FL 32310 USA (e-mail: dixon@magnet.fsu.edu; adkins@magnet.fsu.edu; bird@magnet.fsu.edu; bole@magnet.fsu.edu; toth@magnet.fsu.edu).

H. Ehmler, M. Hoffman, and P. Smeibidl are with the Helmholtz-Zentrum Berlin, 14109 Berlin, Germany (e-mail: hartmut.ehmler@helmholtz-berlin.de; matthias.hoffman@helmholtz-berlin.de; peter.smeibidl@helmholtz-berlin.de).

Color versions of one or more of the figures in this paper are available online at http://ieeexplore.ieee.org.

Digital Object Identifier 10.1109/TASC.2014.2361098
TABLE I

Hybrid Magnet Operating System Parameters

\begin{tabular}{cc}
\hline \hline Central field & $25 \mathrm{~T}$ \\
$\mathrm{~B}_{0}$, resistive / supercon. & $12 \mathrm{~T} / 13 \mathrm{~T}$ \\
Warm bore (mid-plane) & $50 \mathrm{~mm}$ \\
Current & $20 \mathrm{kA}$ \\
Stored energy & $43.6 \mathrm{MJ}$ \\
Ramp time & $30 \mathrm{~min}$ \\
Inductance, resistive / supercon. & $3.8 \mathrm{mH} / 197.1 \mathrm{mH}$ \\
Total scattering angle & $30^{\circ}$ \\
\hline \hline
\end{tabular}

allow for reflection of neutrons upstream and downstream of the beamline toward detectors. The superconducting coil is a 13-T, 600-mm bore coil consisting of $\mathrm{Nb}_{3} \mathrm{Sn} / \mathrm{CICC}$ and weighs 5 ton (6.5 ton full cold mass).

Fabrication of the superconducting cable-in-conduit coil and full cold-mass assembly was completed at the National High Magnetic Field Laboratory and then was transported to Criotec Impianti in Italy where Paschen and leak tests were conducted as part of acceptance qualifications and the major components of the cryostat were assembled around it. Subsequently, the coil with cryostat was transported to a temporary "magnet assembly" hall at the HZB (shown in Fig. 1) then rotated to have the magnet axis horizontal and the remaining aspects of the cryostat assembled. After magnet commissioning the system will be moved to its final location in the neighboring neutron guide hall. Features of the magnet system and details of its assembly are presented with an emphasis on the cold-mass and cryostat.

\section{Hybrid MAGnet System Description}

A sectional view of the magnet system is pictured in Fig. 2. The resistive coils have a conically shaped inner diameter to conform to the bore. It is series connected to a single superconducting coil with $\mathrm{Nb}_{3} \mathrm{Sn} \mathrm{CICC}$ conductor. The entire magnet system has the bore horizontal so it can align with the neutron beam axis. In addition, the magnet system will sit on a platform so it can rotate $+/-15^{\circ}$ for increased exposure of samples to the neutron beam.

All cryogenic and electrical utilities port through an upper "turret" for interface with the house utilities. The thermal shields are cooled via the helium refrigerator at nominally $50 \mathrm{~K}$.

A large part of the structural requirements of the cryostat stem from the electromagnetic interactions between the superconducting and resistive coils. Features are designed to accommodate potential axial and radial misalignments and axially offsetting fault forces created from a burn-out of the resistive coils. 


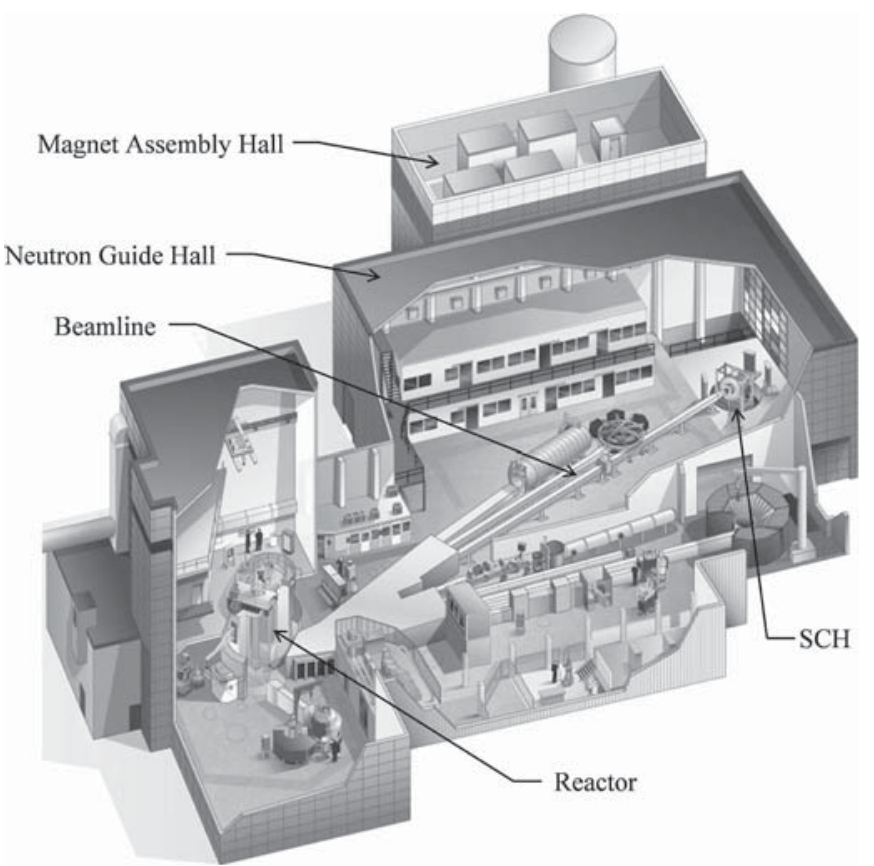

Fig. 1. Neutron guide hall and magnet assembly hall at the HelmholtzZentrum Berlin with the SCH pictured in its operational location.

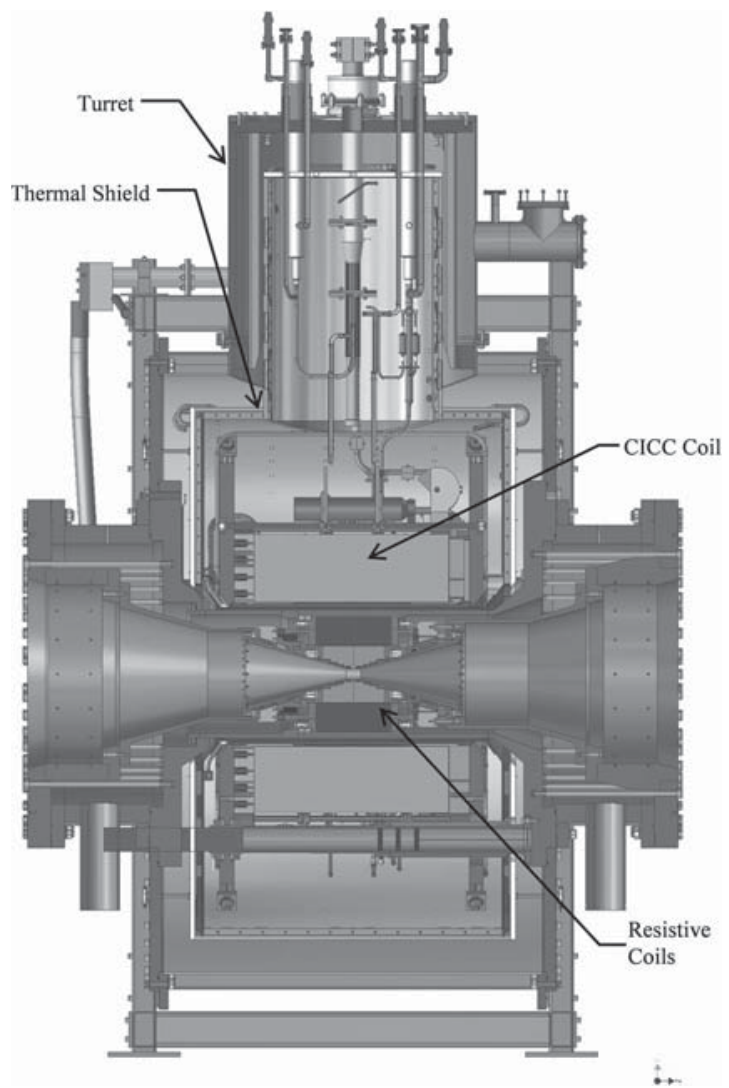

Fig. 2. SCH Outsert Coil with resistive insert coils positioned in the cryostat. The cryogenic and electrical utilities enter through the upper turret.

\section{A. Fault Forces}

Resistive magnets at the NHMFL have a limited lifetime and are usually replaced prior to the coils internally shorting out through continual monitoring of the deviation in resistance. On occasion however, shorts can develop that change the current

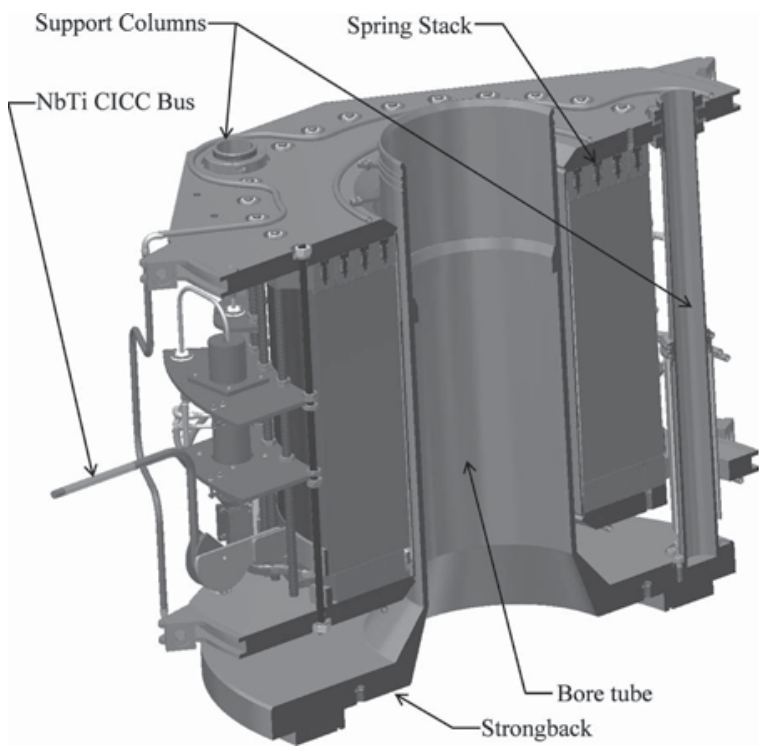

Fig. 3. Isometric and sectioned view of the cold mass positioned on three support columns attached to the strongback/bore tube.

path within the coil, which creates a shift in its magnetic center, for a short period of time until the protection system discharges the magnet. Although unlikely, the worse-case scenario occurs if one half of the resistive coils, above or below the midplane, shorts out. In this case the recentering force for the HZB $\mathrm{SCH}$ can reach 1.12 MN. To accommodate this, a significant supporting structure is put into place to carry the load path between the superconducting and resistive coil. This consists of a "strongback" and three columns, shown in Fig. 3.

The strongback is part of the cryostat end cap which is $95 \mathrm{~mm}$ thick and bridges the load between the two coil types. The columns are tapered stainless steel tubes linking the strongback to the cold mass. The taper takes advantage of the higher strength at the lower temperature end to reduce the overall heat load.

\section{B. Radial Interaction}

The attractive force between the superconducting and resistive cols in the radial direction is $6 \mathrm{kN} / \mathrm{mm}$ where the distance is the displacement from concentricity. While the support columns are stiff in the axial direction, they are much too compliant radially to be effective. Thus for this, titanium warmto-cold links are utilized that connect the cold mass to the shell of the cryostat. These links (4 at each end of the cold mass) are shown in Fig. 4. In addition to the radial forces, the lower links help support the weight of the cold mass.

\section{Axial Interaction}

During normal operation, the axial force interaction can potentially shift the superconducting coil. To prevent this the coils are intentionally offset $2 \mathrm{~mm}$, while warm so that a $1.1 \mathrm{~mm}$ offset exists while cold and operating. The axial electromagnetic stiffness is $12 \mathrm{kN} / \mathrm{mm}$ between the superconducting and resistive coils. Thus ideally the coil sets are attracted to each other by $13.2 \mathrm{kN}$. The load path goes through the bore tube and strongback. 


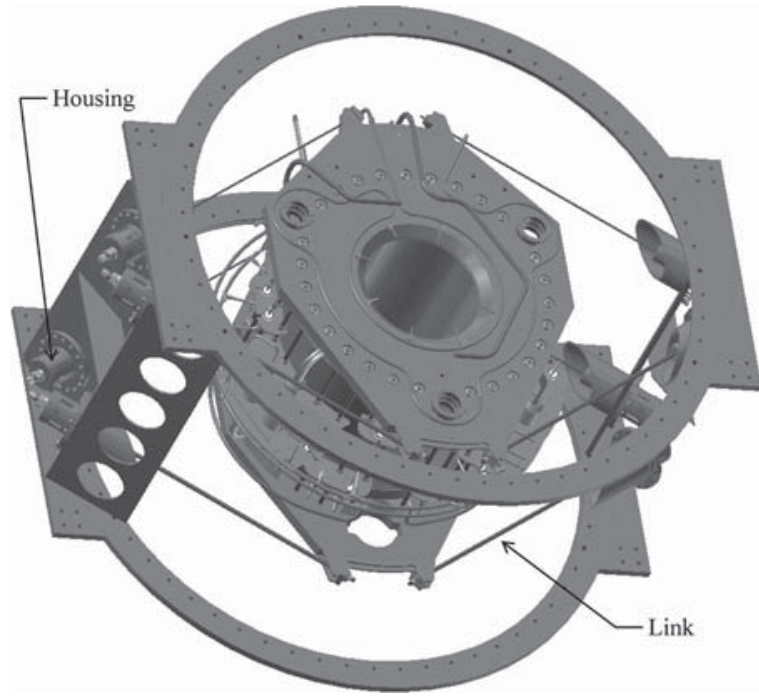

Fig. 4. Warm-to-cold links connecting the cold mass to the cryostat. A link and housing cross-section is shown in the inset.

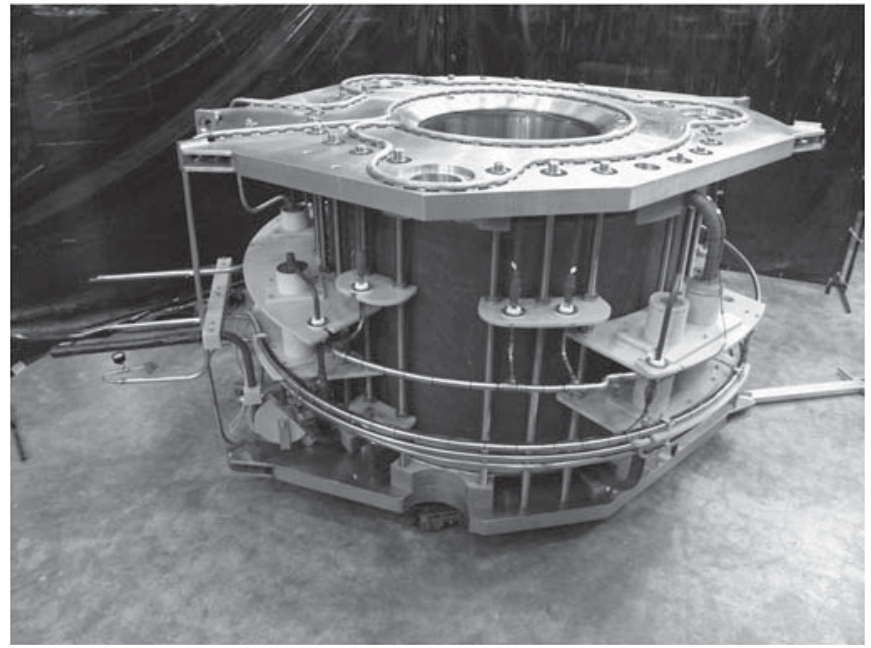

Fig. 5. Photo of the assembled cold mass at the NHMFL.

In addition, a network of Belleville (spring) washers are used to maintain a preload between the superconducting coil and its "ground" flange, counteracting errors during assembly and tolerance stack-up. The springs reside in pockets placed in G-10 end fillers that are adhered to one end of the coil. During assembly of the cold mass, the coil is sandwiched between end flanges and with several sets of springs placed between one coil end and its end flange, as shown in Fig. 3. The assembly is prestressed with a hydraulic actuator to $250 \mathrm{kN}$, with $5 \mathrm{~mm}$ of spring travel, and then the flanges are locked in place via the thin, welded bore tube and tie rods located about the outer diameter. The springs allow an estimated $110 \mathrm{kN}$ of compression to continue as a gap develops between the coil and end flange when the coil shrinks from cool-down and electromagnetic loading.

\section{SyStem ASSEMBLY}

\section{A. Cold Mass}

Being internally cooled, the superconducting CICC coil does not reside in a helium bath. The coil is however supported by a frame, pictured in Fig. 5 and described in the previous section.

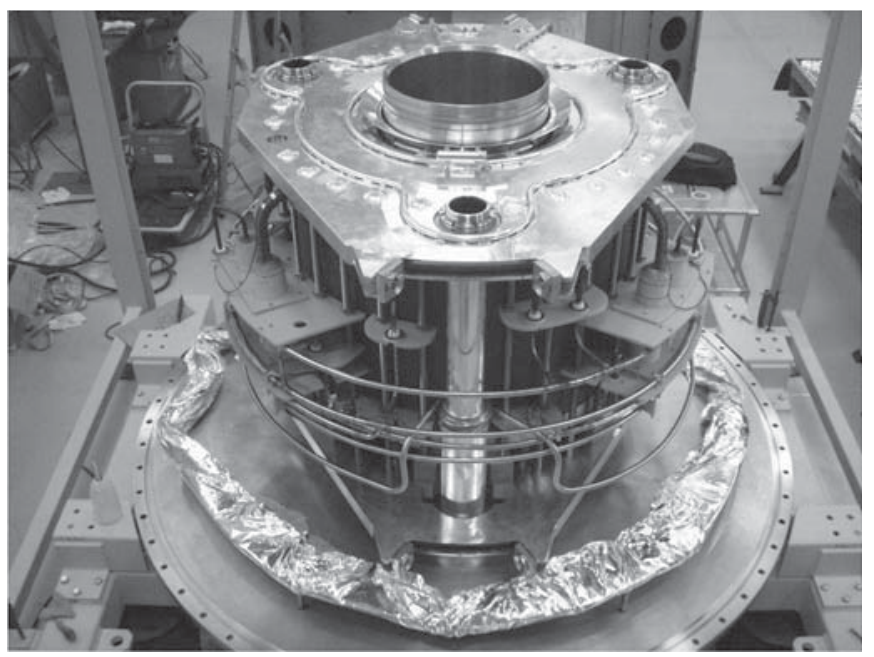

Fig. 6. Cold mass positioned onto the three support columns, strongback, and end plate of the cryostat.

The vacuum environment however creates the risk of a Paschen condition, which is an electrical breakdown that occurs more readily in gasses at low pressure [4], during quench. Paschen tests of the full coil were conducted and described in [5].

Helium plumbing lines are shown that encircle the coil. Supercritical helium is force-flowed into each layer, entering one end and exiting the opposing end. Two parallel helium circuits are used (layers 1-7 and layers 8-18) and individually valve controlled to allow for the ability to throttle helium during cool-down [6]. Voltage isolation of the plumbing circuit is provided by ceramic isolators. The end flanges of the coil are traced with plumbing that connect to the outlet flow of the coil to aid in cool-down.

The splice and termination joints are fully insulated with epoxy-glass and encased in G-10 tubes. The joint supports restrain the relative radial displacements but allow axial motion. Each splice pair are welded together and insulated.

$\mathrm{The} \mathrm{Nb}_{3} \mathrm{Sn}$ termination joints connect to a $\mathrm{NbTi}$ bus that interfaces to the HTS leads. The warm-to-cold link attachment points are located at the coil end plates.

\section{B. Initial Cryostat Assembly}

The early stages of the cryostat assembly took place at the vendor's facility at Criotec Impianti, Italy. Acceptance Paschen test (to $3.5 \mathrm{kV}$ ) and helium leak tests (15 bar and under $1 \times 10^{-8} \mathrm{mbar} \cdot 1 / \mathrm{s}$ ) were conducted for the cold mass. The work performed at Criotec included the major assembly of the cryostat components, attachment of the cold mass to the support columns, temperature sensor installation, and connection of the cryostat plumbing to the cold mass plumbing.

After the coil was placed on the support columns (Fig. 6) the field on axis was mapped to confirm the location of the central field. Once MLI was applied over the thermal shields, the outer vacuum vessel and end plate were attached and the system was readied for transport to $\mathrm{HZB}$.

\section{Final Cryostat Assembly}

The first significant task performed at HZB on the assembly was to deploy the warm-to-cold links. This was done prior to 


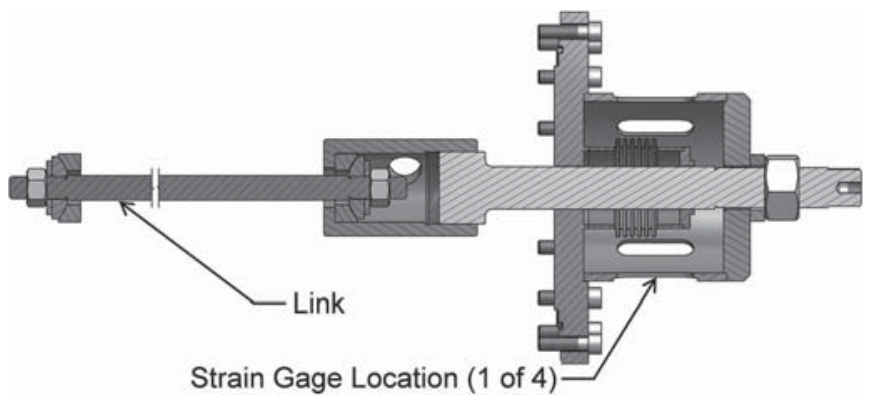

Fig. 7. Warm-to-cold link (with link shortened for clarity) showing location of strain gages on thinned wall of housing.

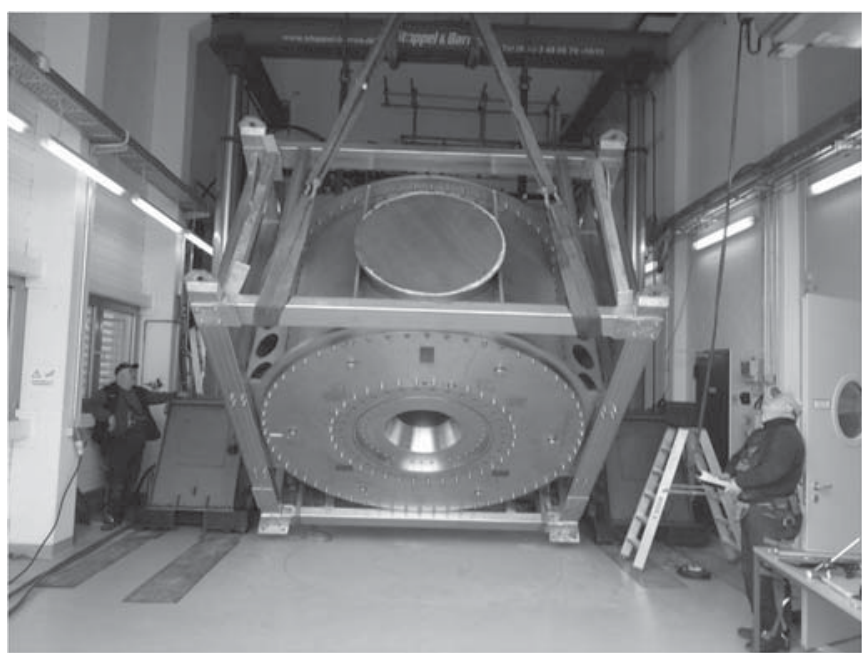

Fig. 8. Rotation of the magnet system to orient the magnet axis horizontal, in the magnet assembly hall.

rotation of the system (to have the magnet axis horizontal) because it was never intended to support the cold-mass in a cantilevered fashion with only the three support columns.

To track the tension of the links, and thus coil centering, strain gages with thermal compensation [7] were attached to the housings, as shown in Fig. 7. Tensioning of the links places the housing in compression. The walls of the housings were machined thinner to increase the sensitivity. Prior to the housing installation, a calibration of each was done to know the forcestrain relation. Once the housings were attached to the cryostat and links connected, each link was initially adjusted to $10 \mathrm{kN}$. After the magnet system was rotated (see Fig. 8) the load in the now upper links decreased and lower links increased. This indicated that the columns deflected slightly placing the cold mass off-center with respect to the warm bore/resistive magnet housing. To regain concentricity, the upper links were adjusted to read $10 \mathrm{kN}$ again. The load on the lower links equated to the preload plus the cold mass weight.

The final assembly details included installation and soldering of the HTS leads [8] (the insulated interface was Paschen tested to $3 \mathrm{kV}$ and down to 0.13 mbar utilizing a small, local vacuum chamber), connection of the instrumentation and helium plumbing, and construction of the upper turret. The last major component included installation of the resistive coils and their magnet housing, shown in Fig. 9.

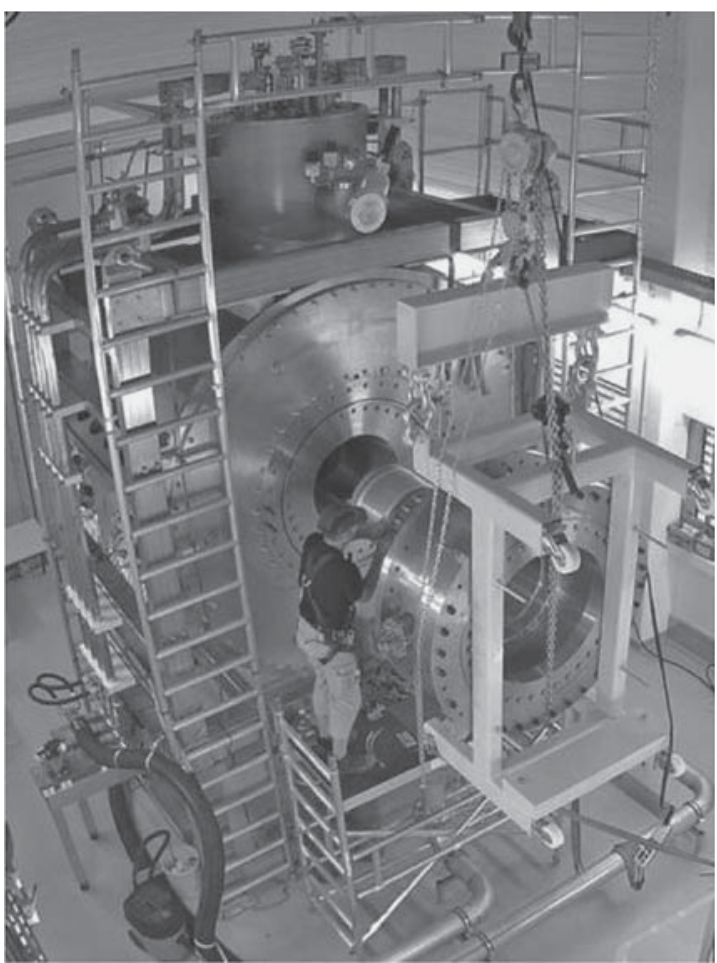

Fig. 9. Resistive magnet installation. The resistive coils are attached to their magnet housing which connects to the face of the cryostat.

\section{CONCLUSION}

The 25-T Series-Connected Hybrid for neutron science at the Helmholtz-Zentrum Berlin will mark a significant improvement in available field strength with large scattering angles. The cold mass has been integrated with the cryostat and interfaced with the house utilities. In addition the resistive magnet has been installed. Commissioning will occur in a temporary "magnet assembly" building. Once completed, the system will be relocated to the neighboring neutron guide hall for science operations.

\section{REFERENCES}

[1] M. D. Bird et al., "The NHMFL hybrid magnet projects," IEEE Trans. Appl. Supercond., vol. 19, no. 3, pp. 1612-1616, Jun. 2009.

[2] P. Smeibidl, A. Tennant, H. Ehmler, and M. D. Bird, "Neutron scattering at highest magnetic fields at the Helmholtz Centre Berlin," J. Low Temp. Phys., vol. 159, no. 1/2, pp. 402-405, Apr. 2010.

[3] M. D. Bird et al., "The powered scattering-magnet program at the NHMFL," IEEE Trans. Appl. Supercond., vol. 18, no. 2, pp. 525-528, Jun. 2008.

[4] J. Knaster and R. Penco, "Paschen tests in superconducting coils: Why and how," IEEE Trans. Appl. Supercond., vol. 22, no. 3, Jun. 2012, Art. ID. 9002904.

[5] I. R. Dixon, T. A. Adkins, H. Ehmler, W. S. Marshall, and M. D. Bird, "Reaction heat treatment and epoxy impregnation of a large $\mathrm{Nb}_{3} \mathrm{Sn} \mathrm{CICC}$ coil for a series-connected hybrid magnet," IEEE Trans. Appl. Supercond., vol. 24, no. 3, Jun. 2014, Art. ID. 4300505.

[6] H. Bai, M. D. Bird, K. R. Cantrell, I. R. Dixon, and A. V. Gavrilin, "Design of cryogenic system for SCH magnets," IEEE Trans. Appl. Supercond., vol. 19, no. 3, pp. 1596-1599, Jun. 2009.

[7] D. M. McRae and R. W. Walsh, "Retrofit of load sensors on $\mathrm{SCH}$ prototype link outer housing," Nat. High Magn. Field Lab., Tallahassee, FL, USA, NHMFL Internal Rep. MDC-13-022, Nov. 15, 2013.

[8] R. Wesche et al., "Results of the test of a pair of $20 \mathrm{kA}$ HTS current leads," J. Phys., Conf. Ser., vol. 507, no. 3, 2014, Art. ID. 032056. 